\title{
Understanding the role of echocardiography in patients with obstructive sleep apnea and right ventricular subclinical myocardial dysfunction - comparison with other conditions affecting RV deformation
}

\author{
Ioana Maria Chetan'1, Bianca Domokos Gergely ${ }^{2}$, Adriana Albu ${ }^{3}$, Raluca Tomoaia ${ }^{1}$, Doina \\ Adina Todea ${ }^{2}$
}

${ }^{1}$ Heart Institute "N. Stancioiu", ${ }^{2}$ Pulmonology Department, ${ }^{3} 2^{\text {nd }}$ Internal Medicine Department, "Iuliu Hatieganu" University of Medicine and Pharmacy Cluj Napoca, Romania

\begin{abstract}
Despite efforts to treat obstructive sleep apnea syndrome (OSA), the condition remains an important risk factor in the development of cardiovascular disease. Early detection of RV dysfunction with novel echocardiographic techniques (speckle tracking echocardiography) may be useful in preventing progression to pulmonary hypertension, with subsequent heart failure and cardiovascular death. Echocardiography is the method of choice for the evaluation of OSA consequences on the heart. Although standard echocardiographic parameters are routinely used in these patients, there are several limitations in the early detection of RV dysfunction. The main concerns are the complex geometry of RV and the impact of pre- and afterload on RV myocardium, which cannot be assessed through standard measurements. The aim of this review is to highlight the utility of advanced echocardiographic parameters in the identification of OSA patients with subclinical myocardial dysfunction, which are at risk of developing heart failure and later adverse events. Speckle tracking echocardiography might provide higher sensitivity in unmasking alterations in RV function when conventional echocardiographic methods cannot detect them. Therefore, this method has a major role in the detection of early stages of RV dysfunction, along with better risk stratification and better timing in the initiation of therapy.
\end{abstract}

Keywords: obstructive sleep apnea; speckle tracking; echocardiography

\section{Introduction}

Obstructive sleep apnea (OSA) is a potentially dangerous sleep disorder, with an incidence of about $4-7 \%$ in the general adult population [1]. It is characterized by the presence of intermittent episodes of partial or complete obstruction of the upper airways during sleep, leading to intermittent hypoxemia, fragmentation of sleep, snoring and daytime sleepiness. Obesity is an important risk fac-

Received 13.07.2020 Accepted 15.10.2020

Med Ultrason

2021, Vol. 23, No 2, 213-219

Corresponding author: Bianca Domokos Gergely

"Leon Daniello" Pneumology Hospital,

6 BP Hasdeu street,

400332 Cluj-Napoca, Romania

Phone: +40741499968

E-mail: biancadomokos@yahoo.com tor, alongside smoking, alcohol consumption and male sex. The hallmark of OSA is the craniofacial morphology, with its contribution to upper airway collapsibility [2]. Some of these risk factors for OSA are also established risk factors for cardiovascular diseases, making it difficult to demonstrate a causal relationship between OSA cardiac damage independent of these potentially confounding factors [3].

In order to diagnose OSA, sleep studies (polysomnography) are required; these can quantify the apnea-hypopnea index (AHI), which places OSA in a severity class (mild, moderate and severe, respectively 5-14, 15-30 and $>30$ events/hour) [4]. A large number of epidemiological evidences describes a link between OSA syndrome and cardiovascular morbidity and mortality $[1,4,5]$, evidence that has prompted the American Heart Association (AHA) to issue a scientific statement describing the need 
to recognize this pathology as an important target for cardiovascular disease risk reduction therapy [5]. Front-line treatment of OSA implies stabilizing the upper airway mechanically, by introducing a column of air via continuous positive airway pressure (CPAP). Although CPAP is considered the "gold standard" treatment, its results are narrowed by poor patient adherence to, due to various side effects [6]. Several forms of cardiovascular diseases (CVD) including stroke, heart failure, hypertension, coronary artery disease, arrhythmias (atrial fibrillation), have been associated with OSA [7]. However, the exact effect of OSA on cardiac structure and function is not fully understood [8]. Previous reports have shown that pulmonary hypertension ( $\mathrm{PH}$ ) occurs in up to $40 \%$ of patients with OSA along with heart or pulmonary disease, but it may also develop in OSA patients in the absence of known cardiopulmonary disorders. Although $\mathrm{PH}$ is mild to moderate in OSA, it can lead to RV hypertrophy and dysfunction. [9]. Podszus et al [10] found that OSA is a predisposing factor for the development of pulmonary hypertension, but there are still few and discrepant data concerning the repercussions of OSA on the structural and functional changes of the right heart [11].

A comprehensive echocardiography screening for subclinical heart failure in OSA patients is needed. It is well known that assessment of the right ventricle (RV) by echocardiography in day-to-day practice is often based on qualitative evaluation, thus being recognized as the "forgotten chamber" [12]. It is important to obtain a complete assessment of the RV, quantitative as well as qualitative, using combined techniques. Threedimensional echocardiography (3DE) and speckle tracking echocardiography (STE) allow the evaluator to overcome the limitations of conventional echocardiography.

The aim of this review was to highlight the utility of advanced echocardiography parameters in identifying OSA patients with subclinical myocardial dysfunction, along with better risk stratification and well-timed initiation of therapy.

\section{Is $3 D$ echocardiography a reality of daily clinical practice when assessing the $R V$ ?}

Most conventional methods of echocardiographic evaluation of the RV function and dimensions are based on approximate volumetric measurements. Such approaches are limited by the complex geometry of the $\mathrm{RV}$ and by their angle or load dependency. As a rule, the problem of the complex geometry of the RV could be solved through real time 3DE and that of the angle/ load dependence through STE (fig 1). Compared to bidimensional echocardiography (2DE), the real time 3DE allows a better anatomical definition of the RV, with good highlighting of the base, apex and outflow tract $[11,13]$.

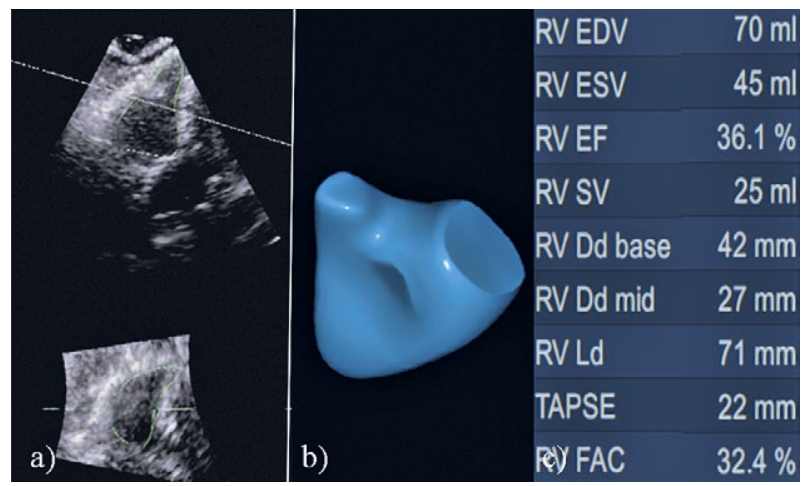

Fig 1. a) Representative right ventricular (RV) quantification using EchoPAC software. After tracing the endocardial border at end-diastole and end-systole b) 3D reconstruction of the RV was automatically realized; c) 3D RV ejection fraction (3D RV $\mathrm{EF})$ and 3D RV fractional area change (32.4\%) in a patient with moderate obstructive sleep apnea. 3D-RVEF=36.1\%. EDV indicates end-diastolic volume; EF, ejection fraction; ESV, endsystolic volume; RV, right ventricular; RVEF, RV ejection fraction; SV, stroke volume; TAPSE, tricuspid annular plane systolic excursion; FAC, fractional area change; Dd, diameter.

Novel techniques using software based on RV 3DE acquisitions have proven the utility to accurately and reproducibly assess the RV dimensions and function [14]. A first study to assess 3DE derived global and regional RV shape parameters in normal subjects and in patients with $\mathrm{PH}$ was developed by Addetia et al [15]. The authors showed that in $\mathrm{PH}, \mathrm{RV}$ expresses modifications in regional curvature evident in apical and septal free-wall regions and in the outflow tract, when compared to normal RV. In this study, the outflow tract was found to be rounder, with more convex apical and body portions of the septum, bulging into the left ventricle (LV) at enddiastole and end-systole, while the apical free wall was found to be more flattened. These findings demonstrate that curvature analysis using $3 \mathrm{DE}$ allows a better quantitative assessment of RV remodeling, due to the tracking of changes in the regional RV shape [15]. The feasibility, accuracy, and reproducibility of RV volumes and ejection fraction (EF) assessment using real-time 3D echocardiographic imaging were pointed out in several studies. For example, the value of 3DE in the evaluation of RV structure and function in patients with congenital heart disease (CHD) was assessed by van der Zwaan et al [16] who used cardiac magnetic resonance imaging (CMR) as a reference. They have compared 3DE derived RV volumes and EF with 2DE derived measurements for RV assessment and demonstrated that 3DE showed a higher specificity to exclude RV myocardial dysfunction. These findings are in agreement with other published studies. Moreover, Nagata et al showed that in 446 patients, 3DE RVEF was independently corelated with cardiac outcomes 
in subjects with diverse backgrounds. Furthermore, 3DE RV-EF offered additional values over clinical risk factors and other echocardiographic parameters, in prediction of future cardiovascular adverse outcomes [17].

Other advantages of 3DE are the relatively short time required for off-line analysis of acquisitions and the good quality of 3D images used for reconstructions, which were demonstrated by the study of Tamborini et el [18]. Several previous studies have proven the reproducibility and fidelity of 3DE in the evaluation of RV systolic function and global volume [19-20]. For example, Zhou et al [3] concluded that using real time $3 \mathrm{DE}$ in the assessment of RV systolic function is an important key in the noninvasive evaluation of severity in OSA; furthermore, they have demonstrated patients with OSA, RV global EF assessed by 3DE was lower than in controls with an inverse relationship to the severity of OSA as assessed by AHI. Similar results were obtained by Vitarelli et al [21], who demonstrated that RVEF assessed by 3DE was lower in subjects with moderate-sever OSA compared with controls, irrespective of the presence of PH. Moreover, the authors have concluded that changes in parameters were independently associated with AHI. Interestingly, after using CPAP for 4 months, the subgroup with severe OSA showed a significantly lower pulmonary artery pressure than that of patients at baseline and an improvement in $\mathrm{RV}$ dysfunction with an increase of RV EF.

It is well known that CMR represents the gold standard in terms of quantitative assessment of the RV; however, its common use in daily practice is limited because it is expensive, time-consuming and sometimes contraindicated. In a number of studies, the accuracy of $3 \mathrm{DE}$ was compared with CMR, and they were in good concordance [22]. Jenkins et al confirmed that 3DE has less variation and higher correlation to CMR than all $2 \mathrm{DE}$ techniques, when being compared in patients after acute myocardial infarction [23]. Also, when comparing 3DE and 2DE with CMR, 3DE EF was the most reliable echocardiographic variable for recognizing CMR-derived RV EF $<50 \%$ [24]. On the other hand, Kjaergaard et al [25], found at best modest correlations between $3 \mathrm{DE}$ and CMR, concluding that tricuspid annular plane systolic excursion (TAPSE) is the best option for RV function assessment for daily clinical purposes. However, it is important to keep in mind that TAPSE assesses only longitudinal RV shortening, which does not represent the RV global function, especially if extensive regional abnormalities are present [26].

In order to use 3DE for RV assessment it is important to remember that the RV cavity borders can be difficult to define, given the numerous trabeculations of RV free wall. This may be the explanation for the differences between 3DE and CMR in the evaluation of RV function and volumes and also the reason why there are cases when RV chamber is underestimated using 3DE [27].

\section{Speckle tracking echocardiography}

in the evaluation of the $R V$ :

the future is here, what's next?

Conventional and Tissue-Doppler Echocardiography allow the evaluation of RV function through fractional area change (FAC), myocardial performance index (MPI), TAPSE and lateral S' wave by tissue-Doppler. STE is a relatively new technique used in the evaluation of myocardial function and is less angle independent. As a principle of this method, random noise is filtered out, while preserving unique stable myocardial features, mentioned as speckles. These blocks of speckles can be unmasked within the myocardium based on frameby-frame tracking, simultaneously in several regions of a plan; therefore, the parameters of myocardial function (velocity, strain, strain rate), are being evaluated in the longitudinal, radial and circumferential ventricular axis. Basically, information on both global and segmental myocardial deformation is provided by this method [28]. However, these parameters are limited by load and angle dependency and do not assess all the axis of RV myocardial contractility. Several studies that have used these conventional echocardiographic parameters reported changes in RV function and structure in patients with OSA [29,30]. Moreover, some studies revealed no abnormalities of RV structure and function in OSA patients [31-32]. Thus, it remains a question when the other techniques are necessary, in order to detect more subtle changes in RV deformation, which might be present in patients with OSA. Novel echocardiographic techniques including 2DE and 3DE speckle tracking methods might therefore provide higher sensitivity in unmasking alterations in RV function and structure, in the early stages of RV dysfunction, when conventional echocardiographic methods cannot detect them $[9,33,34]$. These methods proved their utility in the assessment of RV function in acute conditions. For example, Trivedi et al in a retrospective study conducted on patients with pulmonary embolism (PE), demonstrated that RV function assessed by 2D RV free wall strain (FWS) was reduced in PE patients compared with controls. Adding RV FWS to the existing parameters of RV size and function significantly improved the sensitivity and specificity of the diagnosis of PE and might play a role in guiding treatment. [35] More recently Li et al evaluated in 120 patients suffering from COVID-19, the relationship between echocardiography variables (including 2D RV LS) and clinical variables to predict patient prognosis including mortality. RV LS predicted mortality, with a sensitivity and specificity 
of $94.4 \%$ and $64.7 \%$ respectively. The prognostic value of RV LS was better than other indicators of RV function used alone or in combination, and it was maintained in univariate and multivariate Cox regression analysis [36].

\section{DE speckle tracking of the $R V$ \\ in patients with $\mathrm{OSA}$}

RV function plays an important role in the morbidity and mortality of patients with OSA. Early detection of $\mathrm{RV}$ dysfunction before the onset of pulmonary hypertension is crucial in preventing progression to heart failure and cardiac death [37]. This became possible using 2D speckle tracking echocardiography. Studies showed that $\mathrm{RV}$ longitudinal strain had a predicted value in several cardiovascular diseases (CHD, heart failure, valvular heart disease, $\mathrm{PH}$ and OSA). RV strain in OSA patients has not been yet rigorously and exhaustively explored, but there are investigations on this subject with encouraging results. [38]. Recent studies have shown that patients with OSA have shown impairment of global longitudinal strain (GLS-RV) and lateral wall strain (LS-RV) in the presence of preserved RV-EF. As Buonauro et al [39], concluded in their study, OSA patients showed significant reduction in RV GLS $(18.2 \pm 2.4 \%$, $\mathrm{p}<0.001)$ and LS$\mathrm{RV}(17.8 \pm 4.8 \%, \mathrm{p}<0.001)$, but not in septal longitudinal strain (SLS-RV) $(18.8 \pm 3.6 \%$, $\mathrm{p}=0.229)$ compared with healthy controls. RV-GLS correlated with OSA severity, in the absence of important alteration of other echocardiographic parameters of RV systolic function, such as 3DE-EF or TAPSE. Li et al [40] showed significantly reduced RV LS and strain rates of RV apical segments in patients with mild OSA compared to controls, results confirmed by the study of Kepez et al [11]. D'Andrea et al [41], revealed a reduction in both LV and RV GLS $(-13.8 \pm 5.2 \%, \mathrm{p}<0.01)$, as well as RV LS in OSA patients compared with controls. In concordance with these findings, Hammerstingl et al [42], found that RV 2DE GLS $(-14.5 \pm 8.2 \%, \mathrm{p}<0.0001)$ was significantly lower in subjects with higher AHI, compared with controls; and was positively associated with the severity of OSA. Moreover, conventional echocardiography parameters, such as TAPSE, RV MPI, were not significantly impaired in these patients, supposing that they are not sufficiently sensitive in detecting subclinical changes of RV function in OSA.

$\mathrm{RV}$ dysfunction might be the expression of pulmonary hypertension. This hypothesis is supported by several studies, which demonstrate that patients with OSA and right ventricular systolic pressure (RVSP) $\geq 30 \mathrm{mmHg}$, show substantially lower GLS-RV $(18.2 \pm 2.4 \%, \mathrm{p}<0.001)$, LLS-RV $(17.8 \pm 4.8 \%, \mathrm{p}<0.001)$, compared with patients with $\mathrm{RVSP}<30 \mathrm{mmHg}$ (GLS-RV $=22.7 \pm 4.8 \%$, LLS$\mathrm{RV}=25.1 \pm 7.0 \%$ ) [28]. However, these data are in opposition with the results obtained by Altekin et al [37], who did not find any differences in stain or strain rate at patients with OSA and pulmonary hypertension. Concerning RV strain in OSA patients during CPAP therapy, free wall RV strains and global strain improved after CPAP treatment, even in the presence of unchanged conventional parameters [39]. Kim et al showed that CPAP therapy, unlike sham therapy significantly improved RV GLS and also reduced RV dimension [43]. Other data report strain improvement under CPAP therapy in apical segments, but not in the medial or basal ones, explaining why RV global longitudinal strain (average of apical, medial and basal segments) remained unchanged after 6 months of CPAP therapy [42].

\section{$3 D$ speckle tracking in the evaluation of the $R V$ : a new era}

The RV has a complex pattern of contraction, which occurs along three anatomically relevant axes: longitudinal shortening with tricuspid annulus traction towards the apex, anteroposterior shortening by stretching the free wall over the interventricular septum and radial movement of the free wall, also known as the "bellows effect". Although it is well known that the subepicardial layer of the myocardium is rich in circumferential myofibers, in the daily echocardiographic evaluation of the right ventricle, non-longitudinal motion directions are often omitted [44]. Therefore, the mix of deformation marks provides insight into the pathophysiologic mechanics of RV dysfunction. In comparison to 2D STE, the advantage of using 3D STE consists of the possibility of simultaneous assessment of various wall motion indices, in particular: radial strain (RS), longitudinal strain (LS) and circumferential strain (CS) in the entire RV myocardium. Another unique parameter assessed by $3 \mathrm{DE}$ STE is the area change ratio (ACR) or area strain in a regional or entire tracking area [45]. The clinical utility and feasibility of the 3D STE of RV technique were evaluated by Atsumi et al [46], in both an experimental and clinical study, by using sonomicrometry crystals implanted in the RV endocardium, in order to validate 3D STE data. The authors obtained significant correlation between the two techniques. The PH group had significantly lower RV global deformation parameters than the control group. These findings confirm the results of previous studies that used 3D STE modified for RV software, showing that $\mathrm{PH}$ patients have reduced RV strain values, poorer outcomes, lowered $\mathrm{ACR}, \mathrm{LS}$ and CS and increased mortality risk compared with controls [47]. On the other hand, Liu et al evaluated and compared the value of RV LS detected by both 2DE and 3D STE in the risk stratification of patients with pre-capillary PH. Authors demonstrated that $2 \mathrm{D}$ RV LS is better than 3D RV LS for the detections of patients with pre-capillary hypertension intermediate-high risk [48]. 


\section{D speckle tracking echocardiography for the \\ $R V$ : are regional deformation parameters also necessary?}

The myofibers in the myocardium are a complex 3D network, arranged as a multiple helical display with important functional effect. The orientation of these myofibers is important because it helps to understand the pattern of RV contraction in 3D space [42]. Knowing that 3D STE allows a better perception of RV wall dynamic/ motion, the question is which of the $3 \mathrm{D}$ strain parameters is more dependable.

Atsumi et al [46] showed in their experimental study (10 sheep), that RV ACR and RV LS were significantly lower in moderate pulmonary artery banding (peak RV pressure $>40 \mathrm{mmHg}$ ) and in severe pulmonary artery banding (peak RV pressure $>60 \mathrm{mmHg}$ ) compared to baseline values; however, RV CS differed significantly only between baseline and sever PAB, revealing that longitudinal contraction may be more reactive to pressure overload. The difference between LS and CS was explained by the RV myofibers arrangement: the endocardial layer contains longitudinal myofibers, the epicardial layer comprises circumferential myofibers, whereas the epicardial fiber is continuous with the left ventricular myocardium. Therefore, circumferential contraction might be less affected by pressure overload solely in the RV due to its preservation by LV contraction.

On the other hand, in a study comparing 97 patients suffering from PHT with 60 controls, Smith et al [47] demonstrated that all PHT patients had reduced RV strain indices compared with controls; RV AS and CS were strongly correlated to RV EF $(p<0.001)$. LS and RS were also correlated to RV EF, but lesser. Moreover, the authors evaluated mortality at 24 months, showing that RV AS, CS and LS predicted it most accurately. Furthermore, only AS, and in a lesser level, age, were predictors of death, underling the superiority of 3D STE RV - AS over other parameters. A possible explication for why RV CS may be more affected in the group of patients with PHT, is that important structural changes happen during the development of PHT, including a change in fiber orientation and increased wall stress, leading to reduced circumferential contraction (fig 2).

\section{$3 D$ speckle tracking of $R V$ in patients with OSA: \\ future directions}

The impact of OSA on the right ventricular structure and function has been demonstrated, since PAH, impairment of RV systolic function and RV dyssynchrony have an important influence on cardiovascular morbidity and mortality. 3DE speckle tracking allows a better and more comprehensive assessment of the RV wall motion. Moreover, this novel technique is able to detect alterations in

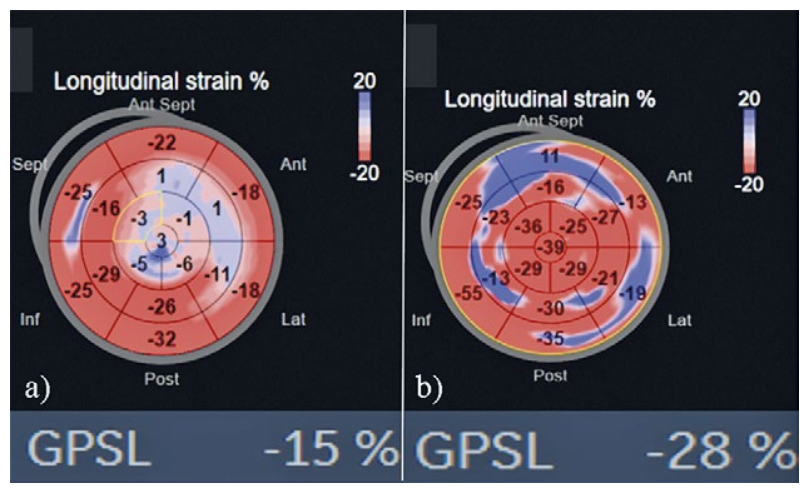

Fig 2. Representative 3D RV strain images in two patients with obstructive sleep apnea. To asses global and longitudinal RV systolic function, we used a 6 segment RV model basal RV free wall, mid RV free wall, apical RV wall, apical septum, mid septum, and basal septum). In both subjects left ventricle function was normal. a) 3D RV speckle-tracking bulls eye in a patient with severe obstructive sleep apnea and PH (decrease of GPSL $R V=-15)$. b) 3D RV speckle-tracking bulls eye in a patient with mild to moderate obstructive sleep apnea without PH (GPSL $\mathrm{RV}=-28$ ). GPSL, global peak longitudinal strain; PH, pulmonary hypertension; $\mathrm{RV}$, right ventricular.

subclinical stages of RV dysfunction. Furthermore, it might be useful in evaluating the efficacy of continuous positive air pressure therapy in improving cardiac function. Thus, an earlier initiation of OSA therapy can reduce cardiovascular risk.

Although these data provide motivation for using the more recent echocardiographic techniques for the assessment of cardiac function in patients with OSA and their response to therapy, several issues need further investigation in order to be accepted as mainstream methods for the quantitative assessment of RV function. Speckle tracking imaging is a more sensitive technique for detecting subclinical alterations in ventricular functions that contrarily may be missed by conventional echocardiography. The favourite deformation parameter is the global longitudinal strain due to its reproducibility and feasibility. 3DE is useful in the determination of ejection fraction, $\mathrm{RV}$ volumes and intracardiac anatomy. It is strongly recommended to develop and validate a dedicated echocardiography program for each specific OSA lesion and to combine deformation imaging with 3DE [49].

\section{Conclusion}

Deterioration of RV mechanics occurs before functional and structural heart damage. Therefore, recent echocardiographic techniques seem to be the cornerstone of detecting subtle cardiac changes in the RV. Since RV demonstrated an important predictive value in several pathologies, it should be more widely used in the evalu- 
ation of patients with OSA, which might present early changes in RV contraction, even in the presence of an apparently preserved systolic function. Risk stratification in these patients using modern echocardiography techniques might bring future information regarding the impact on the cardiac function of the disease and might also offer guidance for early therapy in order to prevent further damage on the systolic RV function.

\section{Conflict of interest: none}

\section{References}

1. Dong R, Dong Z, Liu H, Shi F, Du J. Prevalence, Risk Factors, Outcomes, and Treatment of Obstructive Sleep Apnea in Patients with Cerebrovascular Disease: A Systematic Review. J Stroke Cerebrovasc Dis 2018;27:1471-1480.

2. Hersi AS. Obstructive sleep apnea and cardiac arrhythmias. Ann Thorac Med 2010;5:10.

3. Zhou NW, Pan CZ, Kong DH, et al. A novel method for sensitive determination of subclinical right ventricular systolic dysfunction in patients with obstructive sleep apnea. Clin Respir J 2017;11:951-959.

4. Lee CHK, Leow LC, Song PR, Li H, Ong TH. Acceptance and Adherence to Continuous Positive Airway Pressure Therapy in patients with Obstructive Sleep Apnea (OSA) in a Southeast Asian privately funded healthcare system. Sleep Sci 2017;10:57-63.

5. Bradley TD, Floras JS. Obstructive sleep apnoea and its cardiovascular consequences. Lancet 2009;373:82-93.

6. Broström A, Årestedt KF, Nilsen P, Strömberg A, Ulander M, Svanborg E. The side-effects to CPAP treatment inventory: the development and initial validation of a new tool for the measurement of side-effects to CPAP treatment. J Sleep Res 2010;19:603-611.

7. Shahar E, Whitney CW, Redline S, et al. Sleep-disordered breathing and cardiovascular disease: cross-sectional results of the Sleep Heart Health Study. Am J Respir Crit Care Med 2001;163:19-25.

8. Stradling J, Davies RJ. Sleep apnea and hypertensionwhat a Mess! Sleep 1997;20:789-793.

9. Maripov A, Mamazhakypov A, Sartmyrzaeva M, et al. Right Ventricular Remodeling and Dysfunction in Obstructive Sleep Apnea: A Systematic Review of the Literature and Meta-Analysis. Can Respir J 2017;2017: 1587865.

10. Podszus T, Bauer W, Mayer J, Penzel T, Peter JH, Wichert P. Sleep apnea and pulmonary hypertension. Klin Wochenschr 1986;64:131-134.

11. Kepez A, Niksarlioglu E, Hazirolan T, et al. Early Myocardial Functional Alterations in Patients with Obstructive Sleep Apnea Syndrome. Echocardiography 2009;26:388396.

12. Kossaify A. Echocardiographic Assessment of the Right Ventricle, from the Conventional Approach to Speckle Tracking and Three-Dimensional Imaging, and Insights into the "Right Way" to Explore the Forgotten Chamber. Clin Med Insights Cardiol 2015;9:65-75.
13. Addetia K, Muraru D, Badano LP, Lang RM. New Directions in Right Ventricular Assessment Using 3-Dimensional Echocardiography. JAMA Cardiol 2019;4:936-944.

14. Narang A, Freed B. The Future of Imaging in Pulmonary Hypertension: Better Assessment of Structure, Function, and Flow. Adv Pulm Hypertens 2019;18:126-133.

15. Addetia K, Maffessanti F, Yamat M, et al. Three-dimensional echocardiography-based analysis of right ventricular shape in pulmonary arterial hypertension. Eur Heart J Cardiovasc Imaging 2016;17:564-575.

16. van der Zwaan H, Helbing W, McGhie J, et al. Clinical Value of Real-Time Three-Dimensional Echocardiography for Right Ventricular Quantification in Congenital Heart Disease: Validation With Cardiac Magnetic Resonance Imaging. J Am Soc Echocardiogr 2010;23:134-140.

17. Nagata $\mathrm{Y}, \mathrm{Wu}$ VC, Kado $\mathrm{Y}$, et al. Prognostic Value of Right Ventricular Ejection Fraction Assessed by Transthoracic 3D Echocardiography. Circ Cardiovasc Imaging 2017; 10:e05384.

18. Tamborini G, Brusoni D, Torres Molina JE, et al. Feasibility of a New Generation Three-Dimensional Echocardiography for Right Ventricular Volumetric and Functional Measurements. Am J Cardiol 2008;102:499-505.

19. Renella P, Marx GR, Zhou J, Gauvreau K, Geva T. Feasibility and Reproducibility of Three-Dimensional Echocardiographic Assessment of Right Ventricular Size and Function in Pediatric Patients. J Am Soc Echocardiogr 2014;27:903-910.

20. Lang RM, Badano LP, Mor-Avi V, et al. Recommendations for Cardiac Chamber Quantification by Echocardiography in Adults: An Update from the American Society of Echocardiography and the European Association of Cardiovascular Imaging. J Am Soc Echocardiogr 2015;28:1-39.e14.

21. Vitarelli A, Terzano C, Saponara M, et al. Assessment of Right Ventricular Function in Obstructive Sleep Apnea Syndrome and Effects of Continuous Positive Airway Pressure Therapy: A Pilot Study. Can J Cardiol 2015;31:823831.

22. Shimada YJ, Shiota M, Siegel RJ, Shiota T. Accuracy of Right Ventricular Volumes and Function Determined by Three-Dimensional Echocardiography in Comparison with Magnetic Resonance Imaging: A Meta-Analysis Study. J Am Soc Echocardiogr 2010;23:943-953.

23. Jenkins C, Chan J, Bricknell K, Strudwick M, Marwick TH. Reproducibility of Right Ventricular Volumes and Ejection Fraction Using Real-time Three-Dimensional Echocardiography. Chest 2007;131:1844-1851.

24. Knight DS, Grasso AE, Quail MA, et al. Accuracy and Reproducibility of Right Ventricular Quantification in Patients with Pressure and Volume Overload Using Single-Beat Three-Dimensional Echocardiography. J Am Soc Echocardiogr 2015;28:363-374.

25. Kjaergaard J, Petersen CL, Kjaer A, Schaadt BK, Oh JK, Hassager C. Evaluation of right ventricular volume and function by $2 \mathrm{D}$ and $3 \mathrm{D}$ echocardiography compared to MRI. Eur J Echocardiogr 2006;7:430-438.

26. Morcos P, Vick GW 3rd, Sahn DJ, Jerosch-Herold M, Shurman A, Sheehan FH. Correlation of right ventricular ejec- 
tion fraction and tricuspid annular plane systolic excursion in tetralogy of Fallot by magnetic resonance imaging. Int J Cardiovasc Imaging 2008;25:263-270.

27. Zou H, Leng S, Xi C, et al. Three-dimensional biventricular strains in pulmonary arterial hypertension patients using hyperelastic warping. Comput Methods Programs Biomed 2020;189:105345.

28. Mor-Avi V, Lang RM, Badano LP, et al. Current and evolving echocardiographic techniques for the quantitative evaluation of cardiac mechanics: ASE/EAE consensus statement on methodology and indications endorsed by the Japanese Society of Echocardiography. J Am Soc Echocardiogr 2011;24:277-313.

29. Kasikcioglu HA, Karasulu L, Tartan Z, Kasikcioglu E, Cuhadaroglu C, Cam N. Occult cardiac dysfunction in patients with obstructive sleep apnea syndrome revealed by tissue Doppler imaging. Int J Cardiol 2007;118:203-205.

30. Dursunoğlu N, Dursunoğlu D, Kılıç M. Impact of obstructive sleep apnea on right ventricular global function: sleep apnea and myocardial performance index. Respiration 2005; 72:278-284.

31. Otto ME, Belohlavek M, Romero-Corral A, et al. Comparison of Cardiac Structural and Functional Changes in Obese Otherwise Healthy Adults With Versus Without Obstructive Sleep Apnea. Am J Cardiol 2007;99:1298-1302.

32. Tugcu A, Guzel D, Yildirimturk O, Aytekin S. Evaluation of Right Ventricular Systolic and Diastolic Function in Patients with Newly Diagnosed Obstructive Sleep Apnea Syndrome without Hypertension. Cardiology 2009;113:184-192.

33. Mendoza-Vázquez J, Steiner S, Esquinas AM. Acute and chronic effects of noninvasive ventilation on left and right myocardial function in patients with obstructive sleep apnea syndrome: a speckle tracking echocardiographic study. Echocardiography 2016;33:1623-1624.

34. Mandoli GE, De Carli G, Pastore MC, et al. Right cardiac involvement in lung diseases: a multimodality approach from diagnosis to prognostication. J Intern Med 2020 Sep 30. doi:10.1111/joim.13179.

35. Trivedi SJ, Terluk AD, Kritharides L, et al. Right ventricular speckle tracking strain echocardiography in patients with acute pulmonary embolism. Int J Cardiovasc Imaging 2020;36:865-872.

36. Li Y, Li H, Zhu S, et al. Prognostic Value of Right Ventricular Longitudinal Strain in Patients With COVID-19. JACC Cardiovasc Imaging 2020 Apr 28. doi:10.1016/j. jemg.2020.04.014.

37. Altekin RE, Karakas MS, Yanikoglu A, et al. Determination of right ventricular dysfunction using the speckle tracking echocardiography method in patients with obstructive sleep apnea. Cardiol J 2012;19:130-139.
38. Tadic M, Cuspidi C, Grassi G, Mancia G. Obstructive sleep apnea and cardiac mechanics: how strain could help us? Heart Fail Rev 2020 Feb 3. doi:10.1007/s10741-02009924-0

39. Buonauro A, Galderisi M, Santoro C, et al. Obstructive sleep apnoea and right ventricular function: A combined assessment by speckle tracking and three-dimensional echocardiography. Int J Cardiol 2017;243:544-549.

40. Li J, Wang Z, Li Y, et al. Assessment of regional right ventricular systolic function in patients with obstructive sleep apnea syndrome using velocity vector imaging. Medicine 2016;95:e4788.

41. D'Andrea A, Martone F, Liccardo B, et al. Acute and Chronic Effects of Noninvasive Ventilation on Left and Right Myocardial Function in Patients with Obstructive Sleep Apnea Syndrome: A Speckle Tracking Echocardiographic Study. Echocardiography 2016;33:1144-1155.

42. Hammerstingl C, Schueler R, Wiesen M, et al. Impact of Untreated Obstructive Sleep Apnea on Left and Right Ventricular Myocardial Function and Effects of CPAP Therapy. PLoS One 2013;8:e76352.

43. Kim D, Shim CY, Cho YJ, et al. Continuous Positive Airway Pressure Therapy Restores Cardiac Mechanical Function in Patients With Severe Obstructive Sleep Apnea: A Randomized, Sham-Controlled Study. J Am Soc Echocardiogr 2019;32:826-835.

44. Kovács A, Lakatos B, Tokodi M, Merkely B. Right ventricular mechanical pattern in health and disease: beyond longitudinal shortening. Heart Fail Rev 2019;24:511-520.

45. Jasaityte R, Heyde B, D'hooge J. Current State of ThreeDimensional Myocardial Strain Estimation Using Echocardiography. J Am Soc Echocardiogr 2013;26:15-28.

46. Atsumi A, Seo Y, Ishizu T, et al. Right Ventricular Deformation Analyses Using a Three-Dimensional Speckle-Tracking Echocardiographic System Specialized for the Right Ventricle. J Am Soc Echocardiogr 2016;29:402-411.e2.

47. Smith BC, Dobson G, Dawson D, Charalampopoulos A, Grapsa J, Nihoyannopoulos P. Three-dimensional speckle tracking of the right ventricle. J Am Coll Cardiol 2014;64:41-51.

48. Liu BY, Wu WC, Zeng QX, et al. Comparison of the capability of risk stratification evaluation between two- and threedimensional speckle-tracking strain in pre-capillary pulmonary hypertension. Pulm Circ 2019;9:2045894019894525.

49. Huntgeburth M, Germund I, Geerdink LM, Sreeram N, Udink Ten Cate FEA. Emerging clinical applications of strain imaging and three-dimensional echocardiography for the assessment of ventricular function in adult congenital heart disease. Cardiovasc Diagn Ther 2019;9 (Suppl 2):S326-S345. 